\title{
Association of $A P O E$ polymorphisms and insulin resistance with TCM syndromes in type 2 diabetes patients with macroangiopathy
}

\author{
YI-QIANG XIE ${ }^{1}$, HUA WANG ${ }^{2}$, YUE-PING WU ${ }^{3}$, DE-HUI YIN ${ }^{1}$, \\ ZHUAN-SUO WANG ${ }^{3}$ and YONG-HAO HUANG ${ }^{2}$ \\ ${ }^{1}$ Department of Traditional Chinese Medicine and ${ }^{2}$ Key Laboratory of Tropical Diseases, \\ Hainan Medical College, Haikou 571101; ${ }^{3}$ Department of Endocrinology, Affiliated \\ Hospital of Hainan Medical College, Haikou 571102, Hainan, P.R. China
}

Received April 12, 2011; Accepted July 27, 2011

DOI: $10.3892 / \mathrm{mmr} .2011 .546$

\begin{abstract}
To investigate the association between apolipoprotein $\mathrm{E}(A P O E)$ polymorphisms and insulin resistance and Traditional Chinese Medicine (TCM) syndromes in type 2 diabetes mellitus (T2DM) with macroangiopathy, 60 patients with T2DM macroangiopathy were enrolled and divided into three groups: dryness-heat due to deficiency of yin, Qi-Yin deficiency, and Yin-Yang deficiency, according to the TCM syndromes, with a control group of 20 healthy individuals. $A P O E$ genotype analysis was performed with polymerase chain reaction amplification and restriction fragment length polymorphism, and the results showed that the proportion of the $\varepsilon 4 / 4$ and $\varepsilon 3 / 4$ genotypes and frequencies of the $\varepsilon 4$ and $\varepsilon 3$ alleles were higher in the Qi-Yin deficiency group $(\mathrm{P}<0.05)$. Among the T2DM macroangiopathy patients, the E4 group had the largest number of cases, as well as a significantly longer disease course compared to the $\mathrm{E} 2$ group $(\mathrm{P}<0.05)$. The insulin resistance index (IRI), insulin action index and body mass index (BMI) of patients in the Yin-Yang deficiency group were significantly different from those of patients with dryness-heat due to deficiency of yin and Qi-Yin deficiency. Furthermore, correlation analysis of the BMI and IRI of patients in the Yin-Yang deficiency group revealed a correlation coefficient $r=0.696(\mathrm{P}<0.01)$ and a typical correlation between them. In conclusion, the Qi-Yin deficiency in T2DM patients with macroangiopathy is associated with the $\mathrm{APOE}$ E4 and E3 genotypes. Thus, the APOE gene polymorphism can, to some degree, reflect the TCM syndrome types of T2DM patients with macroangiopathy. Insulin resistance plays an important role in the occurrence of T2DM macroangiopathy and is
\end{abstract}

Correspondence to: Dr Yi-Qiang Xie, Department of Traditional Chinese Medicine, Hainan Medical College, Haikou 571101, Hainan, P.R. China

E-mail: yiqiangxie1972@126.com

Key words: type 2 diabetes mellitus macroangiopathy, apolipoprotein E polymorphism, insulin resistance, Traditional Chinese Medicine syndromes closely associated with the Yin-Yang deficiency according to the TCM differentiating types.

\section{Introduction}

Diabetes mellitus (DM) is a serious disease with great impacts on human life and health. Type 2 diabetes mellitus (T2DM) is the predominant type, accounting for about $90 \%$ of DM cases (1). Chronic complications, especially macrovascular complications, remain difficult to treat, and are the main cause of death and disability in DM patients (2). DM macroangiopathy is a condition in which fat and blood clots stick to and build up in the macrovessels. This disorder affects the heart, brain, and peripheral macrovessels, with atherosclerosis (AS) as its pathological basis (3). However, DM macroangiopathy has a broader area of focus, greater severity, and earlier pathology than pure AS.

Traditional Chinese Medicine (TCM), which encompasses herbal, acupunctural, and osteopathic treatments for disease, has obtained good clinical efficacy in preventing and treating diabetic macroangiopathy through syndrome differentiation. However, few studies have addressed the basis of TCM syndromes, producing an unclear understanding of the clinical syndromes and adding difficulty to diagnosis and treatment. Using the TCM guidelines, T2DM macroangiopathy can be divided into two categories, 'phlegm' and 'blood stasis', according to its pathogenesis (4). The basic pathological features of T2DM macroangiopathy include stasis caused by sputum, a combination of stasis, phlegm and blood deposition in the vessels (5). TCM disease typing for T2DM macroangiopathy hinges on changes or abnormalities in lipid metabolism and lipodogramme expression.

Insulin resistance (IR) is at the heart of T2DM, but is also associated with central obesity, hypertension, dyslipidemia, hypercoagulation tendencies, AS, hyperuricemia, microalbuminuria, and many other diseases (6). Indeed, the common element of these chronic non-traditional progressive diseases is IR. While many other factors have been shown to be involved in IR and diabetes, apolipoprotein $\mathrm{E}$ (APOE) has become a gene of interest in these disorders. APOE, a protein that regulates the uptake and transport of lipoproteins (7), is not only involved in the pathogenesis of AS, but the polymor- 
phisms in the $A P O E$ gene are associated with diabetes and its complications.

In this study, we aimed to determine whether $A P O E$ polymorphisms or IR characteristics are associated with T2DM macroangiopathy. We analyzed allele frequencies of the well-described isoforms of $A P O E,(\mathrm{E} 2, \mathrm{E} 3$ and E4) in patients subdivided according to TCM classifications. We also investigated the association of IR characteristics in these patients.

\section{Materials and methods}

Clinical data. A total of 107 patients with T2DM macroangiopathy visited the Department of Endocrinology at the Affiliated Hospital of Hainan Medical College, Haikou, China from November 2008 to June 2010. Sixty patients were enrolled as experimental subjects, their ages ranging between 48 and 84 years, with a mean age of $(68.33 \pm 9.07)$ years. Another 20 healthy subjects who were free of cardiovascular disease, diabetes, lipid metabolism disorders, endocrine disorders, cancer, and cerebrovascular diseases were enrolled as the controls. The controls ranged in age from 21 to 45 years, with a mean age of $(37.23 \pm 7.23)$ years. Heights and weights of the participants were measured and body mass index (BMI) was calculated as weight $(\mathrm{kg}) /$ height $(\mathrm{m})^{2}$.

Diagnosis of T2DM macroangiopathy was made in accordance with the WHO diagnostic criteria established in 1998 (8). T2DM accompanied by AS of the aorta, coronary artery, basal artery, renal artery, carotid artery, etc., was diagnosed as T2DM macroangiopathy. The TCM syndrome classification criteria were based on the Integrated Traditional and Western Criteria for Diabetes Treatment and Diagnosis (draft) developed by the Diabetes Committee of the Chinese Association of the Integration of Traditional and Western Medicine (9). T2DM macroangiopathy has three differentiating types according to the Clinical Guideline of New Drugs for Traditional Chinese Medicine (10): dryness-heat due to deficiency of yin (primary symptoms: thirst, bulimia, polyphagia, insomnia; secondary symptoms: irritability, heart palpitations, dizziness; tongue: red, with yellow coating; pulse: rapid and forceful), Qi-Yin deficiency (primary symptoms: fatigue, heart palpitations, shortness of breath, dizziness, tinnitus; secondary symptoms: pale face, irritability, insomnia, nocturnal emission, premature ejaculation; tongue: light red, with little coating; pulse: thin and slow), and Yin-Yang deficiency (primary symptoms: drooping spirits, cold limbs, loose stool; secondary symptoms: fatigue, weakness, face swelling, backache, tinnitus; tongue: pale, with white coating, pulse: thin and delayed). T2DM macroangiopathy patients were subdivided into these categories, with 20 patients in each group.

\section{Study methods}

Differentiating methods. Disease history was carefully collected from all participants, and clinical symptoms were identified and evaluated by a self-constructed score scale with 33 possible points. Only those scoring more than 19 points were included, thus 17 of the original 107 patients were excluded based on these scores.

DNA extraction of whole blood. Peripheral blood samples were collected from all participants under fasting conditions. Serum

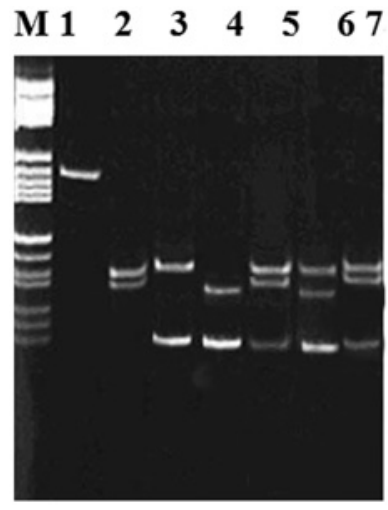

Figure 1. Restriction digestion products for the APOE gene (RFLP-PCR). Lane M, PBR322DNA/Hae III marker; lane 1, PCR amplification products (224 bp); lane 2, 2/2 type produces 2 fragments (91 and $83 \mathrm{bp}$ ); lane 3,3/3 type produces 2 fragments (91 and $48 \mathrm{bp}$ ); lane 4, 4/4 type produces 2 fragments (72 and $48 \mathrm{bp}$ ); lane 5, 2/3 type produces 3 fragments $(91,83$ and $48 \mathrm{bp}$ ); lane 6, 3/4 type produces 3 fragments (91, 72 and $48 \mathrm{bp}$ ); lane 7, 2/4 type produces 3 fragments (91, 83 and $48 \mathrm{bp})$.

$(5 \mathrm{ml})$ was stored at $-20^{\circ} \mathrm{C}$. Serum samples were processed for DNA extraction by adding $0.2 \mathrm{ml}$ EDTA, low-salt buffer (10 mM/1 Tris, $10 \mathrm{mM} / 1 \mathrm{KCL}, 4 \mathrm{mM} / 1 \mathrm{MgCl}_{2}, 2 \mathrm{mM} / 1$ EDTA), and $20 \%$ NP-40. Samples were mixed and centrifuged at $5000 \mathrm{rpm}$ for $5 \mathrm{~min}$ and hemolysates were discarded. Low-salt wash buffer was added 2-3 times to wash white blood cells before the addition of $100 \mu 1$ low-salt buffer and 10\% SDS for incubation at $55^{\circ} \mathrm{C}$ for $5 \mathrm{~min}$. Saturated $\mathrm{NaCl}$ solution $(6 \mathrm{M}$ $\mathrm{NaCl}$ ) was added, followed by centrifugation at $12000 \mathrm{rpm}$ for $5 \mathrm{~min}$. Ethanol was added to precipitate DNA (2.5x supernatant volume). Following centrifugation and washing with ethanol, DNA samples were resuspended in $50 \mu \mathrm{l}$ TE and stored at $-20^{\circ} \mathrm{C}$.

Polymerase chain reaction (PCR) amplification for APOE genotyping. APOE gene PCR primer sequences (Shanghai Bioengineering Technology Service Co., Ltd.) were as follows: upstream primer, 5'-ACAGAATTCGCCCCGGCCTGCTA CAC-3'; downstream primer, 5'-TAAGCTTGGCACGGCTGT CCAAGGA-3'. PCR amplification conditions were as follows: denaturation at $95^{\circ} \mathrm{C}$ for $3 \mathrm{~min} ; 35$ cycles of $95^{\circ} \mathrm{C}$ for $1 \mathrm{~min} \rightarrow$ $59^{\circ} \mathrm{C}$ for $2 \mathrm{~min} \rightarrow 72^{\circ} \mathrm{C}$ for $2 \mathrm{~min} ; 72^{\circ} \mathrm{C}$ for $10 \mathrm{~min}$. PCR products were separated on a $2 \%$ agarose gel to identify a specific band size of $244 \mathrm{bp}$. Restriction digestion of PCR products with Hhal (Shanghai Sangon Corp.) was performed at $37^{\circ} \mathrm{C}$. Digested products were separated on a $12 \%$ polyacrylamide gel for $2 \mathrm{~h}$.

APOE genotyping to identify isoforms. Because three different isoforms exist for $A P O E$, six genotypes are possible following restriction digestion as indicated in Fig. 1. The $\varepsilon 2 / 2$ type produces 91 and $83 \mathrm{bp}$ fragments; the $\varepsilon 3 / 3$ type produces 91 and 48 bp fragments; and the E4/4 type produces 72 and 48 bp fragments. Heterozygous combinations produce the following band patterns: $\varepsilon 2 / 3$ (91, 83 and 48 bp), $\varepsilon 3 / 4$ (91p, 72 and $48 \mathrm{bp}$ ), and $\varepsilon 2 / 4$ (91, 83 and $48 \mathrm{bp}$ ). Allele frequency was determined by the formula $(2 \mathrm{x}$ number of homozygotes + number of heterozygotes $) /(2 \div$ the number of subjects tested $)$. The E2 group comprises $\varepsilon 2 / 2$ and $\varepsilon 2 / 3$ genotypes, the E4 
Table I. Distribution of $A P O E$ genotypes and allele frequencies among TCM syndromes in T2DM macroangiopathy patients.

\begin{tabular}{|c|c|c|c|c|c|c|c|c|c|c|}
\hline \multirow[b]{2}{*}{ Group } & \multirow[b]{2}{*}{$\mathrm{n}$} & \multicolumn{6}{|c|}{ APOE genotype frequency cases $(\%)$} & \multicolumn{3}{|c|}{$\begin{array}{l}A P O E \text { allelic gene } \\
\text { frequency }(\%)\end{array}$} \\
\hline & & $\varepsilon 2 / 2$ & $\varepsilon 2 / 3$ & $\varepsilon 3 / 3$ & $\varepsilon 3 / 4$ & $\varepsilon 2 / 4$ & $\varepsilon 4 / 4$ & $\varepsilon 2$ & $\varepsilon 3$ & $\varepsilon 4$ \\
\hline $\begin{array}{l}\text { Dryness-heat due } \\
\text { to yin deficiency }\end{array}$ & 20 & 0 & $6(30)$ & $4(20)$ & $2(30)$ & $2(0)$ & $6(20)$ & 20 & 40 & 40 \\
\hline Qi-Yin deficiency & 20 & 0 & $3(10)$ & $0(5)^{\mathrm{a}}$ & $9(45)^{\mathrm{a}}$ & $1(5)$ & $7(35)$ & 10 & 30 & $60^{\mathrm{b}}$ \\
\hline Yin-Yang deficiency & 20 & 0 & $4(25)$ & $4(15)$ & $8(40)$ & $1(5)$ & $3(15)$ & 12.5 & 50 & 37.5 \\
\hline Control & 20 & $1(5)$ & $3(15)$ & $8(40)$ & $5(25)$ & $2(10)$ & $1(5)$ & 17.5 & 60 & 22.5 \\
\hline
\end{tabular}

${ }^{a} \mathrm{P}<0.05$ vs. dryness-heat due to yin deficiency; ${ }^{\mathrm{P}}<0.01$, vs. $\varepsilon 3$ and $\varepsilon 2$ alleles in the Qi-Yin deficiency group.

Table II. Relationship between APOE genotypes and age, gender, disease duration, and BMI in T2DM macroangiopathy patients

\begin{tabular}{lccccc}
\hline Characteristics & $\mathrm{n}$ & $\begin{array}{c}\text { Gender } \\
(\text { male/female })\end{array}$ & $\begin{array}{c}\text { Age } \\
(\text { years })\end{array}$ & $\begin{array}{c}\text { BMI } \\
\left(\mathrm{kg} / \mathrm{m}^{2}\right)\end{array}$ & $\begin{array}{c}\text { Disease duration } \\
(\mathrm{years})\end{array}$ \\
\hline Group E2 & 13 & $4 / 9$ & $72.23 \pm 6.61$ & $26.05 \pm 2.60$ & $3.31 \pm 1.75$ \\
Group E3 & 8 & $2 / 6$ & $71.38 \pm 7.43$ & $26.07 \pm 5.88$ & $4.00 \pm 1.60$ \\
Group E4 & 35 & $22 / 13$ & $65.40 \pm 11.85$ & $25.25 \pm 2.93$ & $4.91 \pm 1.50^{\mathrm{a}}$ \\
\hline
\end{tabular}

${ }^{\mathrm{a}} \mathrm{P}<0.05$ vs. E2 group.

group comprises $\varepsilon 3 / 4$ and $\varepsilon 4 / 4$ genotypes, and the E3 group comprises homozygous $\varepsilon 3 / 3$ individuals.

Fasting plasma glucose (FPG) and insulin levels. Fasting serum samples were processed with an automatic biochemical analyzer (Dimension Rxl-Max, German Siemens) using the oxidase method to measure FPG levels. Fasting insulin (FINS) levels were measured using the chemiluminescence method on the Chemiluminescence Immunoassay Analyzer (Beckman Access, Beckman Co.). The IR index (IRI) and insulin action index (IAI) were determined using the following formulas: IRI = FPG x FINS/22.5; IAI = -ln (FPG x FINS) ${ }^{[5]}$. An IRI of $>2.69$ was diagnosed as IR.

Statistical methods. All measurements are expressed as the means \pm standard deviation (SD). A t-test was used to compare the mean values of the multiple samples, and the $\chi^{2}$ test was used to compare the allele frequencies between groups using SPSS13.0 statistical software. The relevance of variables was analyzed by linear regression.

\section{Results}

The association of APOE genotypes with TCM syndromes of T2DM macroangiopathy. The distribution of the genotypes and alleles of APOE are indicated in Table I. The $\varepsilon 3 / 4$ genotype was more common in the Qi-Yin deficiency than in the dryness-heat due to yin deficiency group $\left(\chi^{2}=6.14, p=0.013\right)$, and the $\varepsilon 3 / 3$ genotype was less common in both the dryness- heat due to yin deficiency and the Yin-Yang deficiency group, than in the Qi-Yin deficiency group $\left(\chi^{2}=4.44, \mathrm{p}=0.035\right)$. The $A P O E$ allele frequencies in the Qi-Yin deficiency group were also significantly different $\left(\chi^{2}=11.271, \mathrm{p}=0.004\right)$.

Relationship between APOE alleles and age, gender, disease duration and BMI of T2DM macroangiopathy patients. As shown in Table II, the E4 group comprised the most patients. Females predominated only in the E2 and E3 groups. Additionally, the mean ages of patients decreased slightly from E2 to E3 and from E3 to E4, but were not statistically different. Similarly, BMI was not significantly different between the three groups. However, compared to the E2 group, the E4 group experienced longer disease duration $(\mathrm{t}=-2.671, \mathrm{p}=0.02)$.

BMI, IRI and IAI differ according to TCM syndrome in patients with T2DM macroangiopathy. FINS, IRI, IAI, and BMI are indicated for the TCM categories of our T2DM macroangiopathy patients (Table III). The Yin-Yang deficiency group differed significantly from the other two groups in these characteristics. Furthermore, FBG, FINS and IRI were significantly different between each of the T2DM macroangiopathy groups $(\mathrm{P}<0.01)$ and the control group. Similarly, IAI and BMI were significantly different between each of the T2DM macroangiopathy groups $(\mathrm{P}<0.05)$ and the control group.

The correlation of BMI and IRI of patients in Yin-Yang deficiency group. The correlation between BMI and IRI in 20 
Table III. Comparison of BMI, IRI and IAI in different TCM syndromes for patients with T2DM macroangiopathy ( $\mathrm{n}=20$ per group mean $\pm \mathrm{SD})$.

\begin{tabular}{lccccl}
\hline Group & FBG $(\mathrm{mM} / \mathrm{l})$ & FINS $(\mathrm{uIU} / \mathrm{ml})$ & IRI & IAI & BMI $\left(\mathrm{kg} / \mathrm{m}^{2}\right)$ \\
\hline Dryness-heat due to yin deficiency & $10.26 \pm 3.17^{\mathrm{a}}$ & $14.05 \pm 6.03^{\mathrm{a}}$ & $6.72 \pm 4.42^{\mathrm{a}}$ & $-4.83 \pm 0.62^{\mathrm{b}}$ & $23.95 \pm 2.08^{\mathrm{b}}$ \\
Qi-Yin deficiency & $9.20 \pm 2.48^{\mathrm{a}}$ & $13.07 \pm 5.57^{\mathrm{a}}$ & $5.53 \pm 3.17^{\mathrm{a}}$ & $-4.66 \pm 0.58^{\mathrm{b}}$ & $23.39 \pm 1.75^{\mathrm{b}}$ \\
Yin-Yang deficiency & $10.14 \pm 2.91^{\mathrm{a}}$ & $21.83 \pm 7.88^{\mathrm{a}, \mathrm{c}, \mathrm{d}}$ & $9.63 \pm 3.60^{\mathrm{a}, \mathrm{c}, \mathrm{d}}$ & $-5.31 \pm 0.38^{\mathrm{a}, \mathrm{c}, \mathrm{d}}$ & $29.56 \pm 2.06^{\mathrm{a}, \mathrm{d}, \mathrm{d}}$ \\
Control & $4.87 \pm 0.44$ & $9.18 \pm 3.35$ & $2.03 \pm 0.86$ & $-3.74 \pm 0.40$ & $21.46 \pm 1.74$ \\
\hline
\end{tabular}

${ }^{\mathrm{a}} \mathrm{P}<0.01,{ }^{\mathrm{b}} \mathrm{P}<0.05$ vs. control group. ${ }^{\mathrm{c}} \mathrm{P}<0.01$ vs. dryness-heat due to yin deficiency, ${ }^{\mathrm{d}} \mathrm{P}<0.01, \mathrm{Qi}-$ Yin deficiency groups.

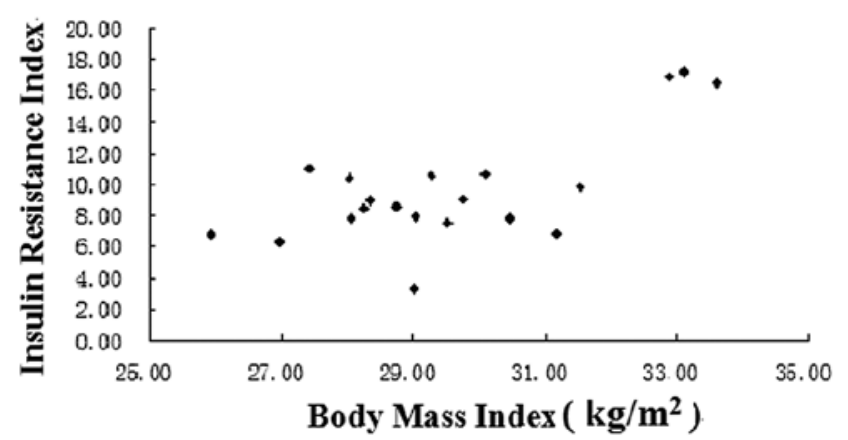

Figure 2. Correlation analysis of BMI and IRI for patients with Yin-Yang deficiency.

patients with Yin-Yang deficiency was analyzed, producing the following linear equation: $\mathrm{y}=25.722+0.994 \mathrm{x}$, with correlation coefficient $\mathrm{r}=0.696(\mathrm{P}<0.01)$; the two indices were confirmed to be related (Fig. 2).

\section{Discussion}

The APOE locus is situated on the long arm of chromosome 19 (19q13). APOE is known to have several isoforms derived from polymorphisms in exon 4 . Thus, the three alleles, $\varepsilon 2, \varepsilon 3$ and $\varepsilon 4$, can produce 6 different genotypes: $\varepsilon 2 / 2, \varepsilon 3 / 3, \varepsilon 4 / 4, \varepsilon 3 / 2$, $\varepsilon 3 / 4$ and $\varepsilon 4 / 2$ (11). The differences between these common isoforms (E2, E3, E4) stems from amino acid substitutions at positions 112 and 158. Arginine at both positions creates the E4 allele, cyseine at both positions creates the E2 allele, and Cys at 112 but Arg at 158 creates the $\varepsilon 3$ allele (12). Our analysis indicates that the $\varepsilon 4$ allele frequency was significantly increased in T2DM patients with macrovascular complications. In contrast, healthy individuals more frequently possessed the $\varepsilon 3$ allele (60\%), similar to reports of allele frequencies in similar healthy populations (13). The amino acid changes between APOE2, APOE3, and APOE4 are enough to reduce the receptor binding activity of $\varepsilon 2$ to only $2 \%$ that of $\varepsilon 3$ (14). Additionally, the metabolic dynamics of $\mathrm{E} 4$ differ from those of E3/E4. Furthermore, total cholesterol and LDL cholesterol levels are higher in people with the $\varepsilon 4$ allele (15). As APOE is involved in immune response and tissue repair, we can conclude that $\varepsilon 4$ has a direct effect on the pathogenesis of AS at the cellular level (16).

These features of $A P O E$ make it a good candidate for involvement in T2DM macroangiopathy. The pathogenesis of this disorder is categorized as either 'phlegm' or 'blood stasis' according to TCM, particulary in that macroangiopathy includes stasis caused by sputum, a combination of stasis and phlegm, and blood deposition in the vessels (4). These different TCM syndromes indicate overall body dysfunction at different levels, and phlegm stasis syndromes have been correlated with $A P O E$ genotypes, which are closely related to lipid metabolism $(17,18)$. Our study indeed indicates that $A P O E$ genotypes are associated with TCM syndromes in T2DM macroangiopathy.

IR plays a critical role in T2DM. Furthermore, hyperinsulinemia during IR can cause vascular disease by promoting synthesis of lipophilic analogs and stimulating the proliferation of smooth muscle cells of the vessel endangium (19). Indeed, high levels of insulin can stimulate synthesis and release of endothelin (ET-1) from endothelial cells, and ET-1 can combine with the endothelin receptor in smooth muscle cells of the endangium to produce strong vasoconstriction (20). In an IR state, vascular endothelial cells can release more platelet-derived growth factor after injury, to stimulate the smooth muscle cells in the tunica media to secrete interleukin-1, tumor necrosis factor, and other cytokines to increase vascular permeability. Thus, more plasma material permeates the inner and medial membranes of the vessel walls, with increased basement membrane components. Insulin-like growth factor secreted by mononuclear cells is thus activated causing increased vascular smooth muscle, fibroblasts, etc., which leads to thickening of the vessel wall (21). IR is closely correlated with vascular dysfunction, disease progression, and elevated blood glucose levels. As a result, decreased sensitivity of the body to insulin will further aggravate the harm of hyperglycemia to the vessels (22).

Our study shows a significant increase in $\varepsilon 3 / 4$ and $\varepsilon 4 / 4$ genotypes in patients with Qi-Yin deficiency, as well as the increased allele frequency of $\varepsilon 4$. At the same time, patients in the $\mathrm{E} 4$ group have a significantly longer disease duration, indicating an internal correlation between TCM syndrome types of T2DM macroangiopathy patients and APOE polymorphism. Thus, APOE genotypes can reflect TCM syndrome types of T2DM macroangiopathy patients to some extent. Additionally, compared to the dryness-heat due to yin deficiency and Qi-Yin deficiency groups, IR was significantly higher in patients with Yin-Yang deficiency. Indeed, insulin can gradually accumulate in the body of T2DM macroangiopathy patients, which, together with the pathological products (phlegm and extravasated blood), can lead to the prolonged duration and aggravation of the disease. 
Yin deficiency causes coldness in the body, leading to further stasis. The Yang Qi is deficient, and therefore, unable to eliminate the coldness; this phenomenon is termed 'stasis caused by deficiency'. Stomach Yin deficiency in the middleburner can cause internal turbid phlegm, which binds together and results in the above disease. Our analysis of BMI and IRI in patients with Yin-Yang deficiency showed a correlation between the two indices, which confirms the relationship between body weight and IR. Because of turbid phlegm, many T2DM macroangiopathy patients have obesity, and obese T2DM macroangiopathy patients frequently have IR. Thus, obese individuals are more likely to have IR, as other studies have also concluded (23). Furthermore, obesity may increase IR. Our study indicates that higher BMI in T2DM macroangiopathy patients is positively related to IRI.

In conclusion, our study shows that TCM syndrome differentiation of T2DM macroangiopathy patients is correlated with both APOE gene polymorphisms and IR. These associations may serve as part of the foundation of the origin of TCM syndromes. Additional research may permit molecular biological methods to diagnose TCM syndromes in T2DM macroangiopathy, providing an important link between TCM and Western Medicine, which may help improve treatment options for these patients.

\section{References}

1. Mensah GA, Mokdad AH, Ford E, Narayan KM, Giles WH, Vinicor F and Deedwania PC: Obesity, metabolic syndrome, and type 2 diabetes: emerging epidemics and their cardiovascular implications. Cardiol Clin 22: 485-504, 2004.

2. Stolar MW: Defining and achieving treatment success in patients with type 2 diabetes mellitus. Mayo Clin Proc 85: S50-S59, 2010

3. Stolar MW: Atherosclerosis in diabetes: the role of hyperinsulinemia. Metabolism 37: 1-9, 1988

4. Wang Q: The present situation of TCM treatment for diabetes and its researches. J Tradit Chin Med 23: 67-73, 2003.

5. Ning G, Hong J, Bi Y, Gu W, Zhang Y, Zhang Z, Huang Y, Wang $\mathrm{W}$ and $\mathrm{Li} \mathrm{X}$ : Progress in diabetes research in China. J Diabetes 1: 163-172, 2009.

6. Bruce KD and Hanson MA: The developmental origins, mechanisms, and implications of metabolic syndrome. J Nutr 140 648-652, 2010 .

7. Greenow K, Pearce NJ and Ramji DP: The key role of apolipoprotein E in atherosclerosis. J Mol Med 83: 329-342, 2005.

8. Alberti KG and Zimmet PZ: Definition, diagnosis and classification of diabetes mellitus and its complications. Part 1: diagnosis and classification of diabetes mellitus provisional report of a WHO consultation. Diabet Med 15: 539-553, 1998.
9. Lan L: Standard for diagnosis and therapeutic effect evaluation of diabetes mellitus by integrative Chinese and Western medicine (draft). Zhongguo Zhong Xi Yi Jie He Za Zhi 25: 94-95, 2005 (in Chinese).

10. Zheng X: TCM New Drug Clinical Research Guidelines. Chinese Medical Science and Technology Press, Beijing, pp1-230, 2002.

11. Davignon J, Gregg RE and Sing CF: Apolipoprotein E polymorphism and atherosclerosis. Arteriosclerosis 8: 1-21, 1988.

12. Huang Y: Mechanisms linking apolipoprotein $\mathrm{E}$ isoforms with cardiovascular and neurological diseases. Curr Opin Lipidol 21: 337-345, 2010

13. Liang S, Pan M, Geng HH, Chen H, Gu LQ, Qin XT, Qian JJ, Zhu JH and Liu CF: Apolipoprotein E polymorphism in normal Han Chinese population: frequency and effect on lipid parameters. Mol Biol Rep 36: 1251-1256, 2009.

14. Arbones-Mainar JM, Johnson LA, Altenburg MK and Maeda N: Differential modulation of diet-induced obesity and adipocyte functionality by human apolipoprotein E3 and E4 in mice. Int J Obes (Lond) 32: 1595-1605, 2008

15. Thakre TP, Mamtani MR and Kulkarni H: Lack of association of the APOE epsilon 4 allele with the risk of obstructive sleep apnea: meta-analysis and meta-regression. Sleep 32: 1507-1511, 2009.

16. Nieminen T, Kahonen $M$ and Lehtimaki T: The effects of apoAI/C-III/A-IV, apoE and apoB polymorphisms on carotid artery intima-media thickness. Future Cardiol 2: 179-186, 2006.

17. Song J and Chen G: Discussion about the relationship of apolipoprotein E and gene polymorphism with TCM Phlegm stasis syndrome. J Trad Chin Med 8: 13-15, 2002.

18. Li L, Li X and Song J: Application of matching matrix, factor analysis and cluster analysis in research on Phlegm stasis syndrome characteristics of coronary heart disease patients. Zhongguo Zhong Xi Yi Jie He Za Zhi 25 4: 343-347, 2006 (in Chinese).

19. Mammi C, Pastore D, Lombardo MF, Ferrelli F, Caprio M, Consoli C, Tesauro M, Gatta L, Fini M, Federici M, Sbraccia P, Donadel G, Bellia A, Rosano GM, Fabbri A and Lauro D: Sildenafil reduces insulin-resistance in human endothelial cells. PLoS One 6: e14542, 2011.

20. Hearly B: Endothelial cells dysfunction: an emerging endocrinopa linked to coronary disease. J Am Coll Cardiol 16: 349-356, 1990.

21. van den Oever IA, Raterman HG, Nurmohamed MT and Simsek S: Endothelial dysfunction, inflammation, and apoptosis in diabetes mellitus. Mediators Inflamm 2010: 792393, 2010.

22. Lee GT and Cho YD: Regulation of fibronectin levels by agmatine and spermine in mesangial cells under high-glucose conditions. Diabetes Res Clin Pract 66: 119-128, 2004.

23. Marette A: Molecular mechanisms of inflammation in obesitylinked insulin resistance. Int J Obes Relat Metab Disord 27 (Suppl 3): S46-S48, 2003. 